\title{
The influence of relative refractive index and core diameter on properties of single- mode optical fiber.
}

\author{
Sama Mumtaz Al-dabbagh ${ }^{1 *}$, Manaf G. Said ${ }^{2}$ \\ 1,2Department of Physics, College of Education for Pure Sciences, University of Mosul, Mosul, Iraq \\ E-mail: ${ }^{1 *}$ sama.m.aldabagh@gmail.com, ${ }^{2}$ manafsaid59@gmail.com
}

(Received April 10, 2020; Accepted July 22, 2020; Available online December 01, 2020)

DOI: 10.33899/edusj.2020.126908.1063, @ 2020, College of Education for Pure Science, University of Mosul. This is an open access article under the CC BY 4.0 license (http://creativecommons.org/licenses/by/4.0/).

\begin{abstract}
:
In this research, the influence of the parameters design, such as the refractive index of the core, the cladding, and the radius of the core on propagation constant $(\beta)$ of single-mode optical fiber in optical communication region (1.2-1.6) $\mu \mathrm{m}$ have been studied and investigated. Material, waveguide, and profile dispersions are analyzed and investigated. Three models of optical fibers with different relative refractive indices $(\Delta)(0.004,0.007,0.01)$ at a wavelength equal $1.55 \mu \mathrm{m}$, and three models of core radius $(3,4,5)$ $\mu \mathrm{m}$ are taken in the count. Numerical simulations and modeling are arranged depending on weakly guiding approximation for solving homogeneous wave equation derived from Maxwell's equations. Our modeling has been solved by the aid of MATLAB software. Material and profile dispersion have no significant change for various relative refractive index, while waveguide dispersion is affected by the change of relative refractive index. the waveguide dispersion increased by increasing core diameter and the profile dispersion decreased as the core diameter increased. There is no effect on martial dispersion by increasing the core diameter.
\end{abstract}

Keyword: Single-mode optical fiber, Propagation constant, Material Waveguide, and profile dispersion.

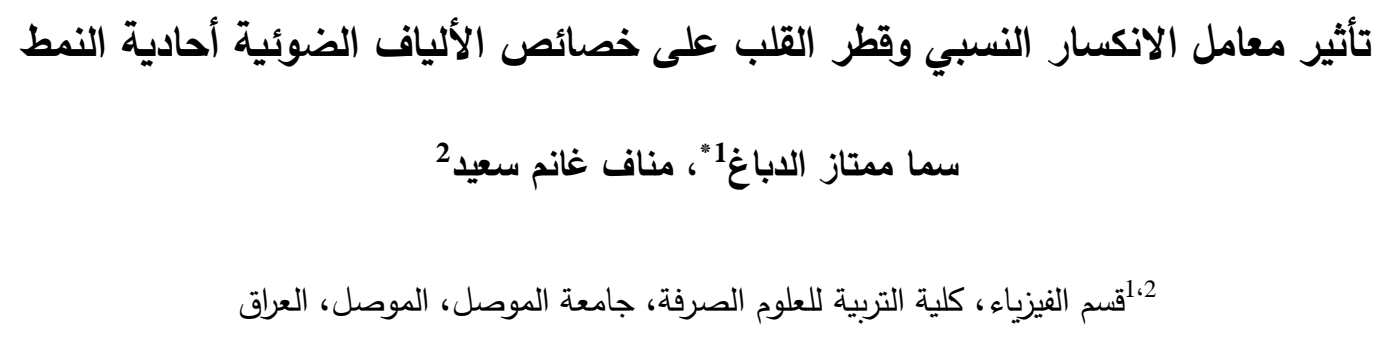

الخلاصة:

دراسة تأثير معلمات التصميم مثل معامل إنكسار القلب والغلاف ونصف قطر القلب لليف الضوئي على ثابت الأنتشار (م) (1)

للألياف الضوئية أحادية النمط في مجال الاتصالات الضوئية بm (1.6-1.2) التي تم إستقصائها في هذا البحث. تم تحليل وتدقيق كل

(0.004, 0.007, من التقزح المادي والموجي والجانبي، لثلاثة نماذج من الألياف الضوئية حسب تغير معامل الانكسار النسبي (ه)

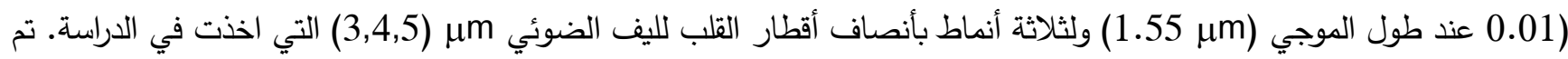

ترتيب المحاكاة العددية والنمذجة بالاعتماد على تقريب التوجيه الضعيف لحل معادلات الموجات المتجانسة المستمدة من معادلات 
ماكسويل. وتمت النمذجة في هذه الدراسة باستخدام برنامج تم اعداده بلغة MATLAB. حيث لاحظنا أن التقزح المادي والجانبي لا يتغير

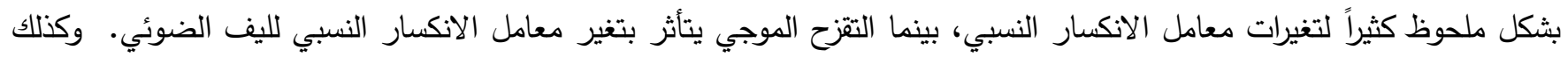

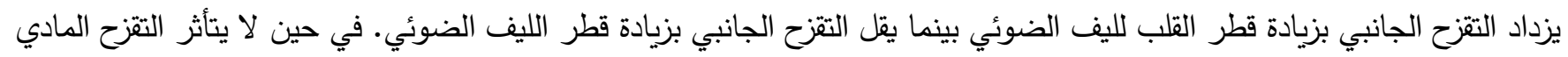
بزيادة قطر القلب لليف الضوئي.

الكلمات الدالة: الالياف الضوئية أحادية النمط، ثابت الانتشار، التثزح المادي والموجي والجانبي.

\section{Introduction}

\section{Step Index}

The step-index fiber is represented by cylindrical waveguide dielectric core that is surrounded by the cladding. This fiber has a core refractive index higher than the cladding refractive index and radius (a). The core and cladding refracting indices are uniform as shown in equation (1).

$\mathrm{n}(\mathrm{r})= \begin{cases}\mathrm{n}_{1} & \text { when } \mathrm{r}<\mathrm{a}(\text { core }) \\ \mathrm{n}_{2} & \text { when } \mathrm{r} \geq \mathrm{a} \text { (cladding) }\end{cases}$

The step-index fiber has two types on the basic model:

- Single-mode step-index.

- Multimode step-index

\section{Single-mode step-index}

Single-mode step-index fiber has a very small central core of diameter which is between (2-10) $\mu m$ , being very small, this diameter leads to one path for light rays through the cable while there is more than one path for light ray in multimode as shown in figure 1.

The difference of refractive index between the layers in fiber is called the relative refraction index $(\Delta)$; it is very small and can be written as in equation (2) [1]:

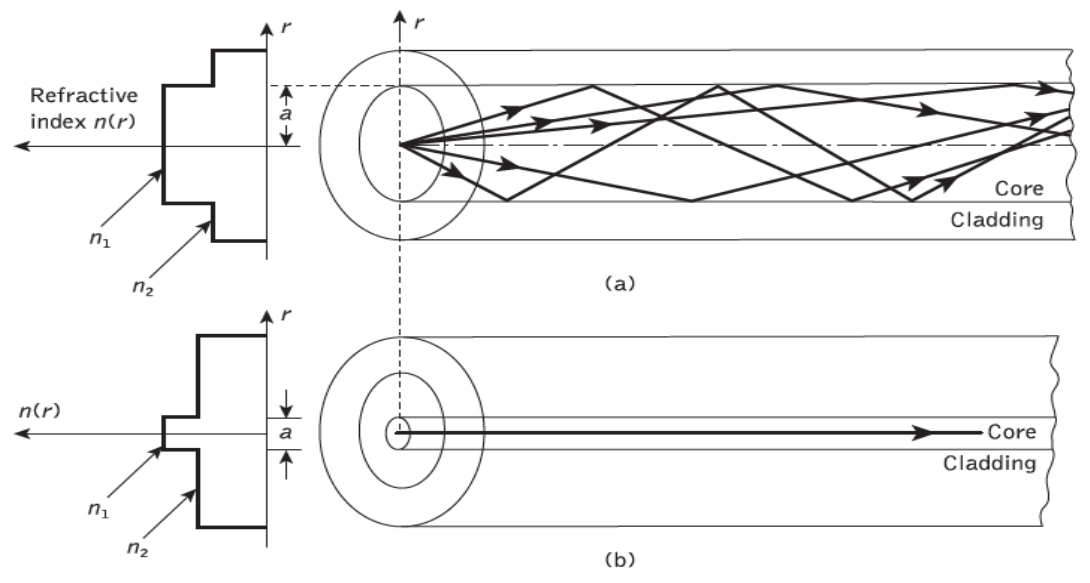

Figure (1) The step-index fiber for (a) multimode step-index fiber; (b) single-mode step-index fiber [2, 3]. 
$\Delta=\frac{n_{1}^{2}-n_{2}^{2}}{2 n_{2}^{2}}$

In weakly guiding fiber $\left(n_{1} \cong n_{2}\right)$, the relative refractive index value:

$\Delta=\frac{n_{1}-n_{2}}{n_{1}}$

For the step-index, the single-mode fiber must be satisfied with the condition $(0<V \leq 2.405)$.

Where $\mathrm{V}$ is Normalized frequency, it is the very beneficial parameter in optical fiber, which briefs all the important characteristics of the fiber in a single number. the normalized frequency used to calculate the number of possible modes and can be used to calculate the cut off wavelength. The relation normalized frequency is given by equation (4).

$V=\frac{2 \pi a}{\lambda} \sqrt{n_{1}^{2}-n_{2}^{2}}$

Where:

a is the core radius, $\lambda$ is the wavelength, $n_{1}$ the refractive index of core and $n_{2}$ the refractive index of the cladding.

The ratio between the angular momentum and the phase velocity is the propagation constant $(\beta)$ of the guided modes; it lies in the values of $\left(n_{2} k<\beta<n_{1} k\right)$, where $k=\frac{2 \pi}{\lambda}$.

To find the Normalized propagation constant (b) [4]

$b=\frac{\left(n_{e f f}^{2}-n_{2}^{2}\right)^{1 / 2}}{\left(n_{1}^{2}-n_{2}^{2}\right)^{1 / 2}}[0,1]$

The change of the fundamental linearly polarized mode $\left(L P_{01}\right)$ propagating along the fiber is defined by the propagation constant $\beta$. It is suitable to define the effective refractive index for single-mode fiber as a ratio propagation constant of the fundamental mode to that of the wavenumber as shown in equation (6) [3].

$n_{\text {eff }}=\frac{\beta}{k}$

Where: $n_{\text {eff }}$ the range between $\mathrm{n}_{1}$ and $\mathrm{n}_{2}$ values.

\section{Dispersion}

In the optical fiber communication system, there are many problems such as dispersion in which the light pulse is spread out when it propagates through the channel of the fiber transmission. The greatest effect of dispersion occurs in the case of digital systems in the form of broadening in the width of transmitter pulses over the fiber; the broadening increased as the traveling distance in the fiber is increased. The negative phenomenon leads to inter symbol interference between traveled pulses and it leads to increase the errors. By increasing the length of the fiber, we get an increase in Dispersion; it is measured by the unit of time per unit of length, such as ns $/ \mathrm{km}, \mathrm{ps} / \mathrm{km}$, or time $/ \mathrm{km}$ [5]. 
In optical fiber communication, the total dispersion parameter is given by:

$D=-\frac{\lambda}{c} \frac{d^{2} n_{e f f}}{d \lambda^{2}}$

where $\mathrm{c}$ is light velocity and $n_{\text {eff }}$ is the effective refractive index.

There are many types of dispersion:

1- Material dispersion

2- Waveguide dispersion

3- Profile dispersion

\section{Material dispersion}

The light emitted from a source with different wavelengths each wavelength propagates with different group velocity in fiber; this occurs due to the change in the refractive index through the fiber material. This effect is called material dispersion [6].

The pulse which is composed of different wavelengths also has the same effect as it propagates through the fiber. This broadening in the pulse depends upon the variation of the refractive index in the fiber along the transmission line [2].

In the dispersive medium, the phase velocity is different from group velocity depending on the amount of the dispersion in this media, when there is no dispersion.

$$
\begin{aligned}
& V_{\text {phase }}=\frac{\omega}{k} \\
& v_{g}=\frac{\delta \omega}{\delta \beta}
\end{aligned}
$$

Where $\omega$ the angular frequency.

The phase and group velocities are the same $V_{g}=V_{p h}$.

The amount of dispersion in the medium depends on the variation of refractive indices through the fiber which tends to spread out or delay the light wave depends on its wavelength; this is called the group velocity delay [4].

The group index shows the way of light behavior when the light pulses are considered the group index that become important because the light pulse broadening in time of the input signals of a different wavelength.

$N \equiv n-\lambda \frac{d n}{d \lambda}$

The plane wave propagated in the core dielectric medium of fiber which refractive index $n(\lambda)$, which is infinitely extended in the direction of propagation, these wave can be used in the evalulation of the dispersion material in the fiber [7].

$D_{m}=\frac{1}{c}\left[\frac{d N_{1}}{d \lambda} A(V)+\frac{d N_{2}}{d \lambda}\langle 1-A(V)\rangle\right]$ 
Where

$A(V) \equiv \frac{1}{2}\left[\frac{d(b V)}{d V}+b\right]$

$D_{m}$ is material dispersion, and $\mathrm{A}(\mathrm{V})$ is the fraction of $\mathrm{L} P_{01}$ mode power that is carried in the core. The total material dispersion in the fiber, which can be considered as sum material dispersion of both core and glad regin.

$$
T_{g}=\frac{1}{c}\left\{N_{1} A(V)+N_{2}[1-A(V)]+N_{2} \Delta[A(V)-b]\right\}
$$

Figure (2), explains the relationship between the material dispersion and wavelength for pure silica. It is observed that the material dispersion tends to zero region around $1.3 \mu \mathrm{m}$.

\section{Waveguide Dispersion: -}

The waveguide dispersion, in single-mode fiber, takes place as the light passes across the core and the cladding because the refractive index in the core is higher than the cladding [8].

The light moved through the core to cladding is more slowly, the difference between the refractive index of the core and the cladding is very small $<<1$.

The different refractive index for the core and the cladding in the single-mode caused the spreading of the light at different speeds and led to the propagation delay; thus the waveguide dispersion.

The chromatic dispersion defined by the combination of the material and waveguide dispersion. [9]

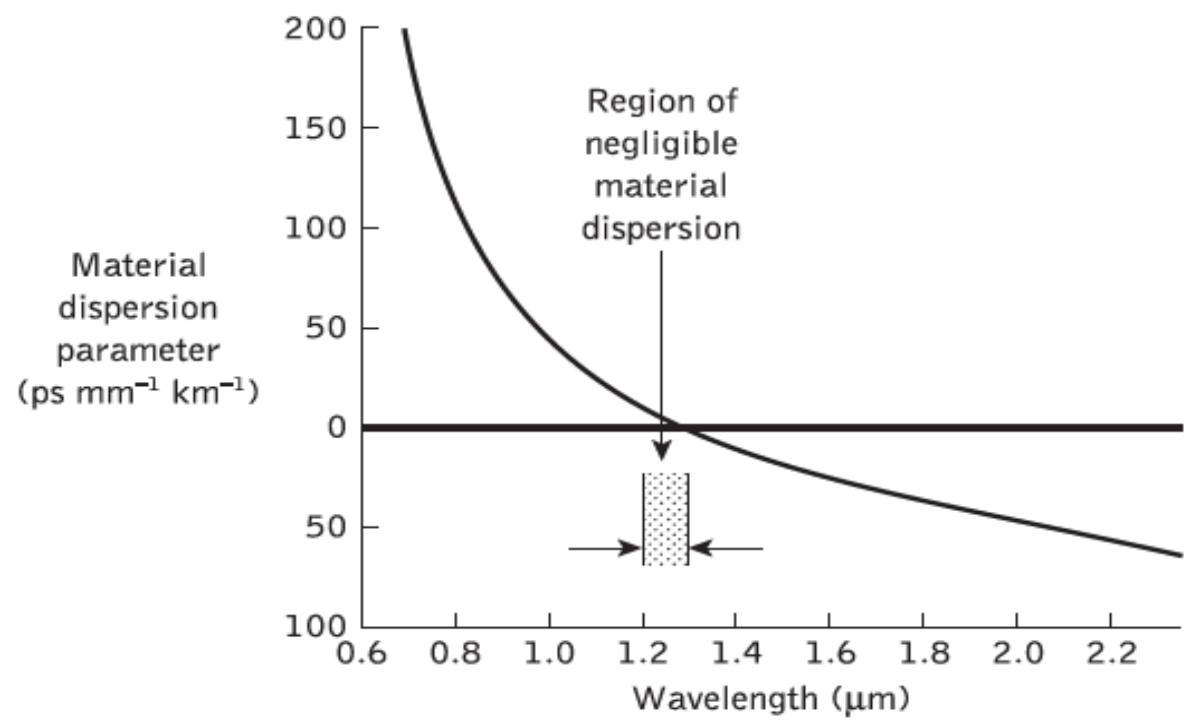

Figure (2). The function between wavelength and material dispersion for silica [2]. 
The material and waveguide dispersion is zero at wavelength $\lambda=1.3 \mu \mathrm{m}$, therefore, the best wavelength in terms of dispersion is $\lambda=1.3 \mu \mathrm{m}$ where is called the zero-dispersion, but the best wavelength in terms of attenuation is $\lambda=1.55 \mu \mathrm{m}$.

The optical fibers' design with the zero-dispersion at a wavelength $\lambda=1.55 \mu \mathrm{m}$ which is called the dispersion-shifted fibers.

\section{Profile dispersion}

The profile dispersion is affected by the refractive index profile and the wavelength of single-mode optical fiber [10].

The determination of the optimum refractive index profile depends on many factors. One of these factors is dispersion that takes place because of the changes in the refractive index between the cladding and the core fiber.

profile dispersion parameter $\mathrm{d} \Delta / \mathrm{d} \lambda$ results can be obtained from calculating the ratio between $\mathrm{d} \Delta$ and $\mathrm{d} \lambda$ for the step-index fiber and multi cladding fiber [2].

The material dispersion cancels the waveguide dispersion and obtained zero total dispersion when the profile dispersion equals zero. Although the profile dispersion is small it is not possible to be neglected with an optical fiber of high numerical aperture. In this paper, then the parameter composite profile dispersion was defined, furthermore, the core influence has been taken in the account, which considers as a good sign in this research. The state of art indicated that the sum of total dispersion can be equal to zero [10].

The profile dispersion is very small (less than $0.5 \mathrm{ps} / \mathrm{km} . \mathrm{nm}$ ), by comparing the two other types of dispersion in the total dispersion components of the multimode case. [11]

$D_{P}=-\frac{N_{2}^{2} \Delta}{n_{2} c \lambda} \frac{y}{2}\left(1+\frac{y}{8}\right)\left[V \frac{d^{2}(b V)}{d V^{2}}+\frac{d(b V)}{d V}-b\right]$

profile dispersion parameter $\mathrm{Y}$ is defined as:

$Y \equiv-\frac{2 n_{2}}{N_{2}} \frac{\lambda}{\Delta} \frac{d \Delta}{d \lambda}$

$D_{T}$ represents the total dispersion in single-mode fiber as shown in equation (15) [7].

$D_{T}=D_{M}+D_{W}+D_{P}$ 


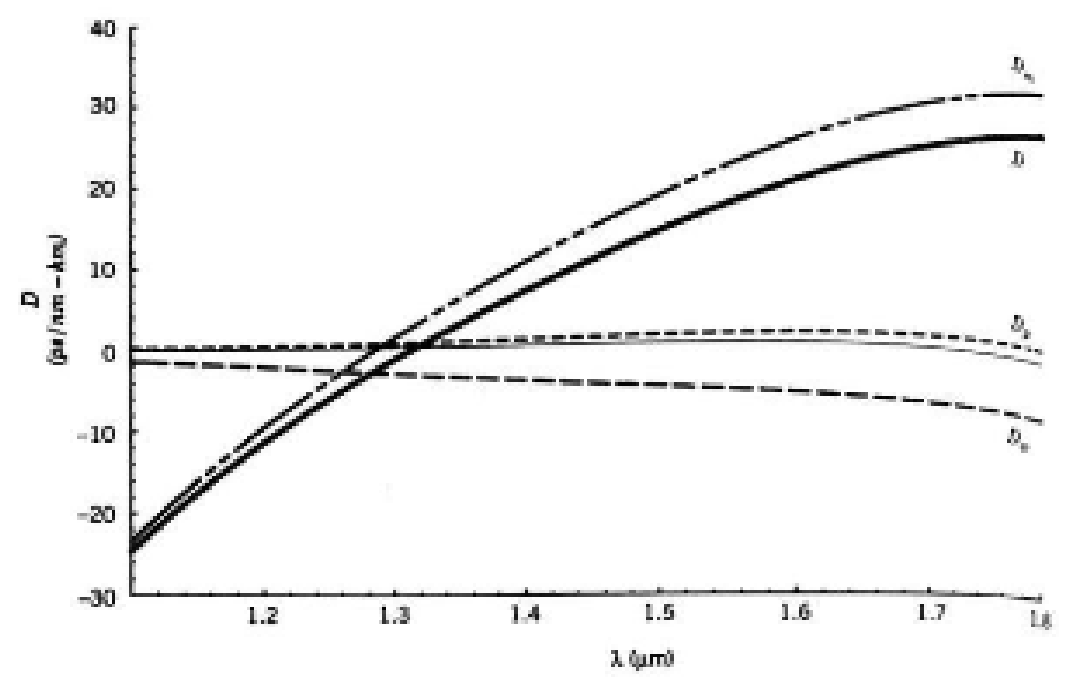

Figure (3) Three types of dispersions and total dispersion properties for conventional and wavelength in single-mode fibers showing the material $\Delta=0.003$ [7].

\section{Results}

1-Table (1) shows the relative refractive indexes for the core and the cladding for the three samples of single-mode optical fibers. by using the Sellmeier formula [12].

$n(\lambda)=\left\{1+\sum_{i=1}^{3} \frac{A_{i} \lambda^{2}}{\lambda^{2}-\lambda_{i}^{2}}\right\}^{1 / 2}$

The relative refractive indexes $(\Delta)$ at wavelength $1.55 \mu \mathrm{m}$ have been considered.

The optional communication range of optical fiber (1.2-1.6) $\mu \mathrm{m}$ has been taken into count. The three optical fibers have a core radius of $4 \mu \mathrm{m}$. 
Journal of Education and Science (ISSN 1812-125X), Vol: 29, No: 4, 2020 (124-139)

Table (1) The Sellmeier parameters used in the papers.

\begin{tabular}{|c|c|c|l|l|l|l|l|}
\hline $\mathbf{K}$ & material & $\mathbf{A}_{1}$ & $\lambda_{1}$ & $\mathbf{A}_{2}$ & $\lambda_{2}$ & $\mathbf{A}_{3}$ & $\lambda_{3}$ \\
\hline $\begin{array}{c}K_{1} \\
(n a)\end{array}$ & $\mathrm{SiO}_{2}$ & 0.6961663 & 0.0684043 & 0.4079426 & 0.1162414 & 0.8974794 & 9.896161 \\
\hline $\begin{array}{c}K_{2} \\
(n c)\end{array}$ & $\mathrm{GeO}_{2}$ & 0.711040 & 0.064270 & 0.451885 & 0.129408 & 0.704048 & 9.425478 \\
\hline $\begin{array}{c}K_{3} \\
(n a)\end{array}$ & $\begin{array}{c}9.1 \% \mathrm{GeO}_{2} \\
83.2 \% \mathrm{SiO}_{2} \\
7.7 \% \mathrm{~B}_{2} \mathrm{O}_{2}\end{array}$ & 0.72393884 & 0.085826532 & 0.41129541 & 0.10705260 & 0.79292034 & 9.3772959 \\
\hline $\begin{array}{c}K_{4} \\
(n c)\end{array}$ & $\begin{array}{c}13.5 \% \mathrm{GeO}_{2} \\
86.5 \% \mathrm{SiO}_{2}\end{array}$ & 0.73454395 & 0.08697693 & 0.42710828 & 0.11195191 & 0.82103399 & 10.846540 \\
\hline $\begin{array}{c}K_{5} \\
(n a)\end{array}$ & $\mathrm{SiO}_{2}$ & 0.6961663 & 0.0684043 & 0.4079426 & 0.1162414 & 0.8974794 & 9.896161 \\
\hline $\begin{array}{c}K_{6} \\
(n c)\end{array}$ & $\begin{array}{c}4.1 \% \mathrm{GeO}_{2} \\
95.9 \% \mathrm{SiO}_{2}\end{array}$ & 0.68671749 & 0.072675189 & 0.43481505 & 0.11514351 & 0.89656582 & 10.002398 \\
\hline
\end{tabular}

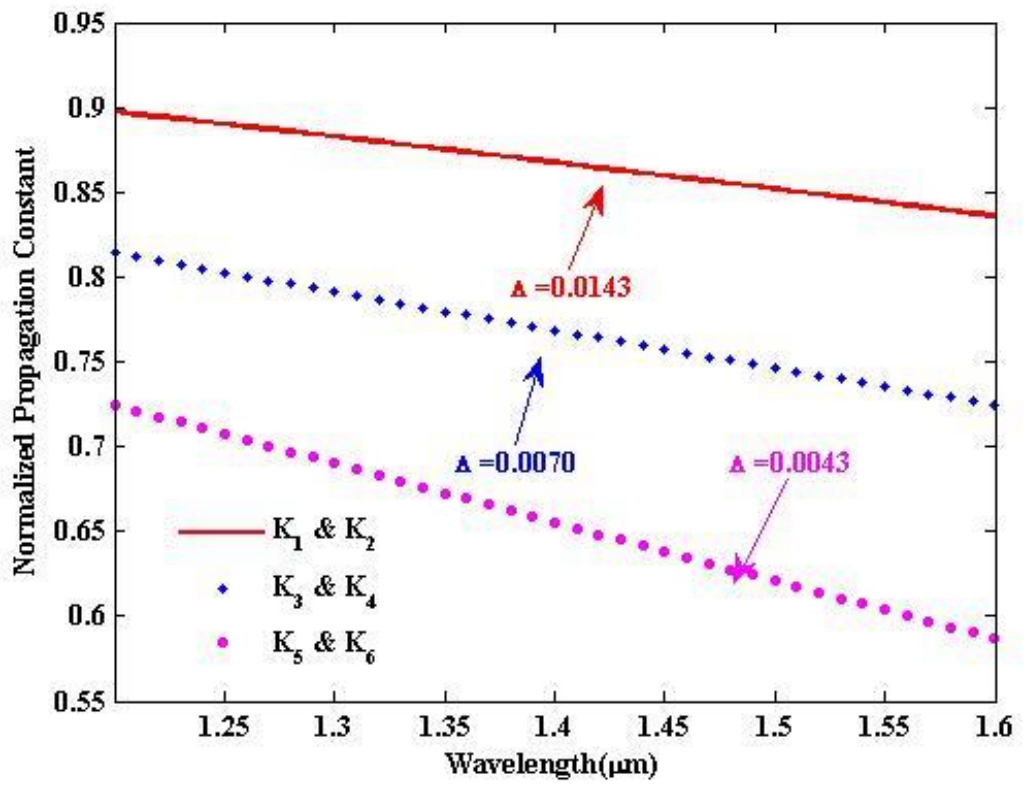

Figure (4) Normalized Propagation constant and wavelength.

The numerical analyses and simulation have been investigated by MATLAB software.

Figure 4 demonstrates the various normalized propagation constant $b$ with wavelength, as relative refractive index $(\Delta)$ increased the normalized propagation constant is increased. In figure 5 by increasing the relative refractive index, there is a shifting of propagation constant toward higher normalized frequency, also, the relation between propagation constant $\mathrm{B}$ and normalized frequency $\mathrm{V}$ is almost linearly. 


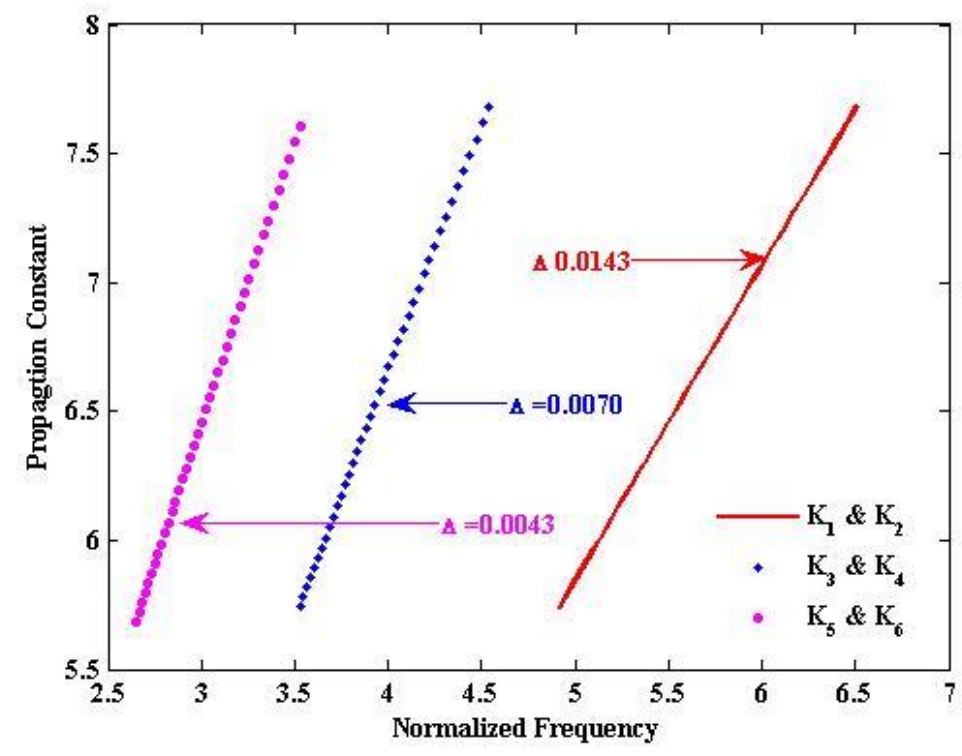

Figure (5) The relation between propagation constant \& normalized frequency.

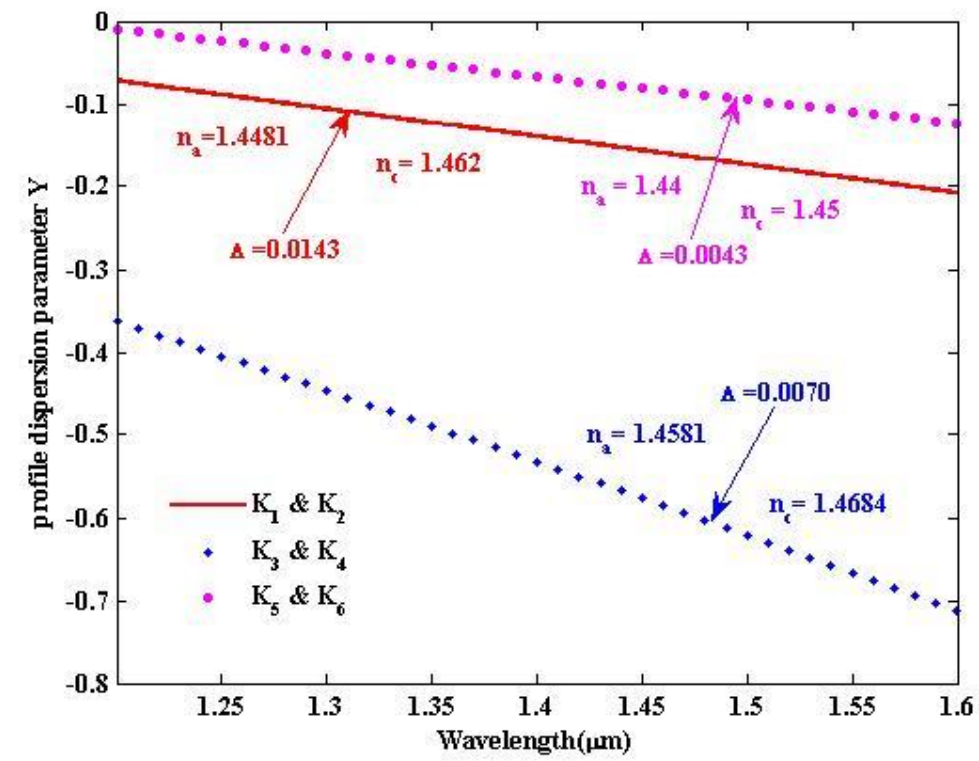

Figure (6) The relation between wavelength $\&$ profile dispersion parameter $y$ in single mode with the relative refractive index.

Figure 6 shows the relation between wavelength and profile dispersion parameter $(\mathrm{Y})$ for fibers constructed from the materials of the table (1), it is noted that the refractive index of the core appears in red $\left(\mathrm{K}_{1} \& \mathrm{~K}_{2}\right)$ has a value (1.462) and the refractive index of cladding equals 1.4481 at $1.55 \mu \mathrm{m}$. The refractive index for core and cladding in blue color $\left(\mathrm{K}_{3} \& \mathrm{~K}_{4}\right)$ has the amount of (1.4684) and 1.4581 respectively. The optical fiber in pink $\left(\mathrm{K}_{5} \& \mathrm{~K}_{6}\right)$ has a refractive index of core equals 1.45 and the refractive index of cladding equals 1.44 , So it is concluded that as the refractive index of the core and 
cladding increases, it leads to decrease in the profile dispersion parameter. In contrast by increasing the wavelength, a decreasing in $\mathrm{Y}$ is noted

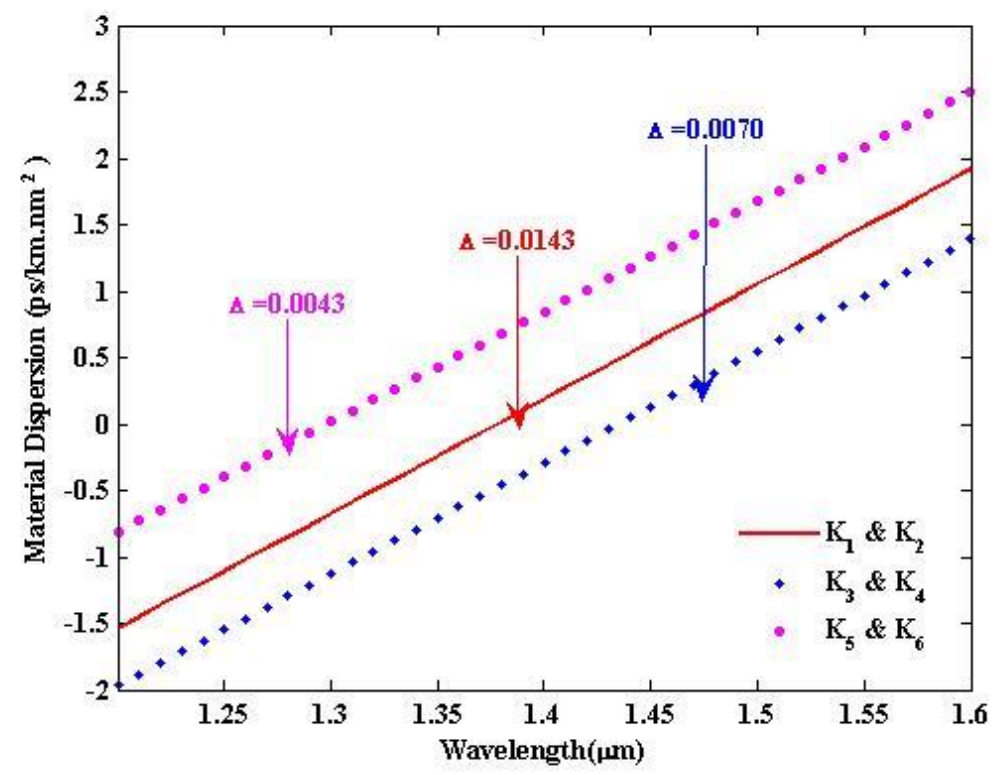

Figure (7) The relation between wavelength \& material dispersion in single-mode with the relative refractive index.

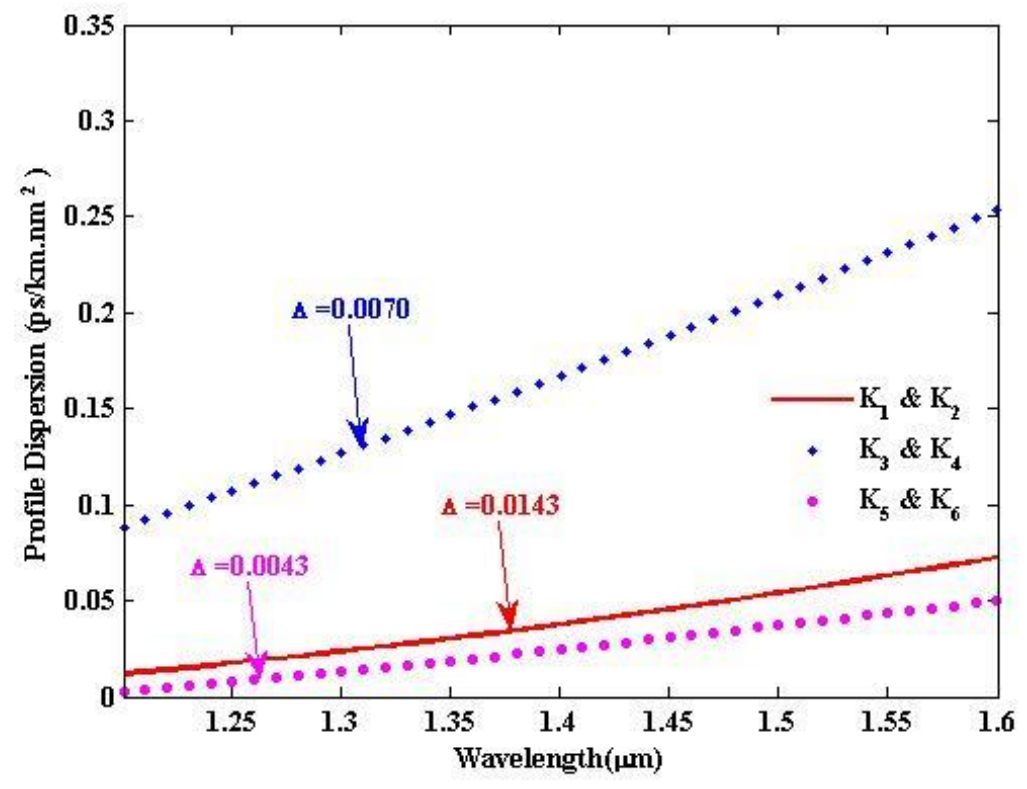

Figure (8) The relation between wavelength $\&$ profile dispersion in single-mode with the relative refractive index.

The material and profile dispersion does not affect the relative refractive index as it is shown in Figures 7 and 8 respectively. In contrast, the waveguide dispersion has increased whenever the relative refractive index increased as shown in Figure 9. 


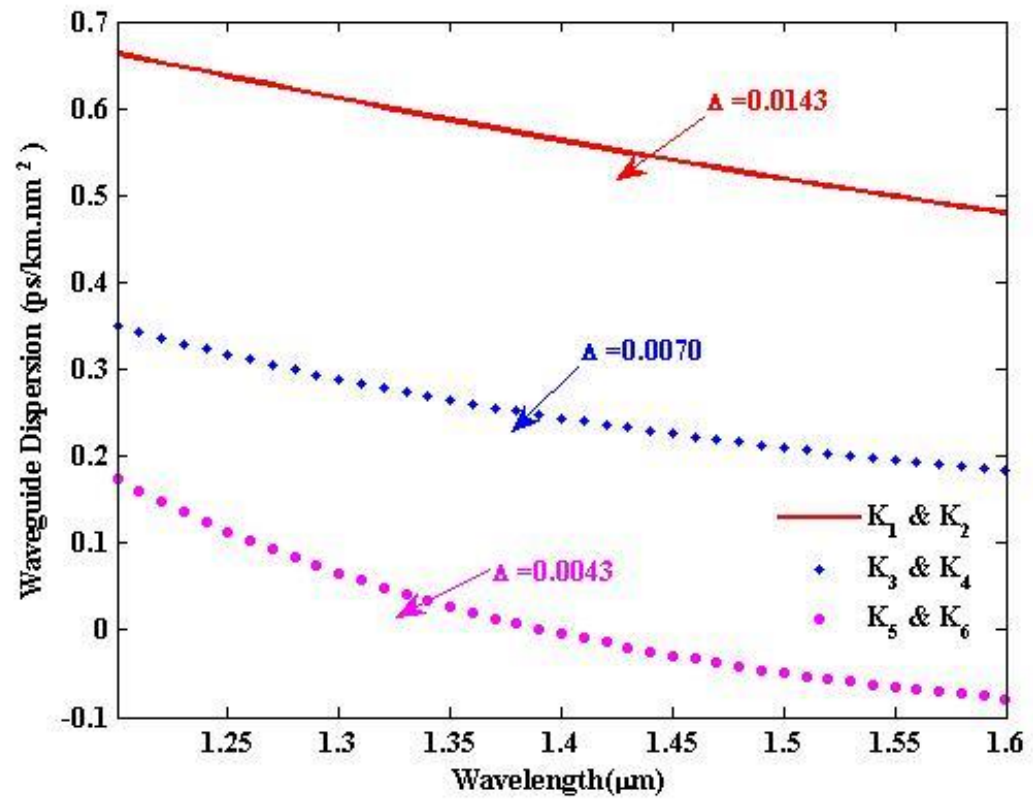

Figure (9) The relation between wavelength $\&$ waveguide dispersion in single-mode with the relative refractive index.

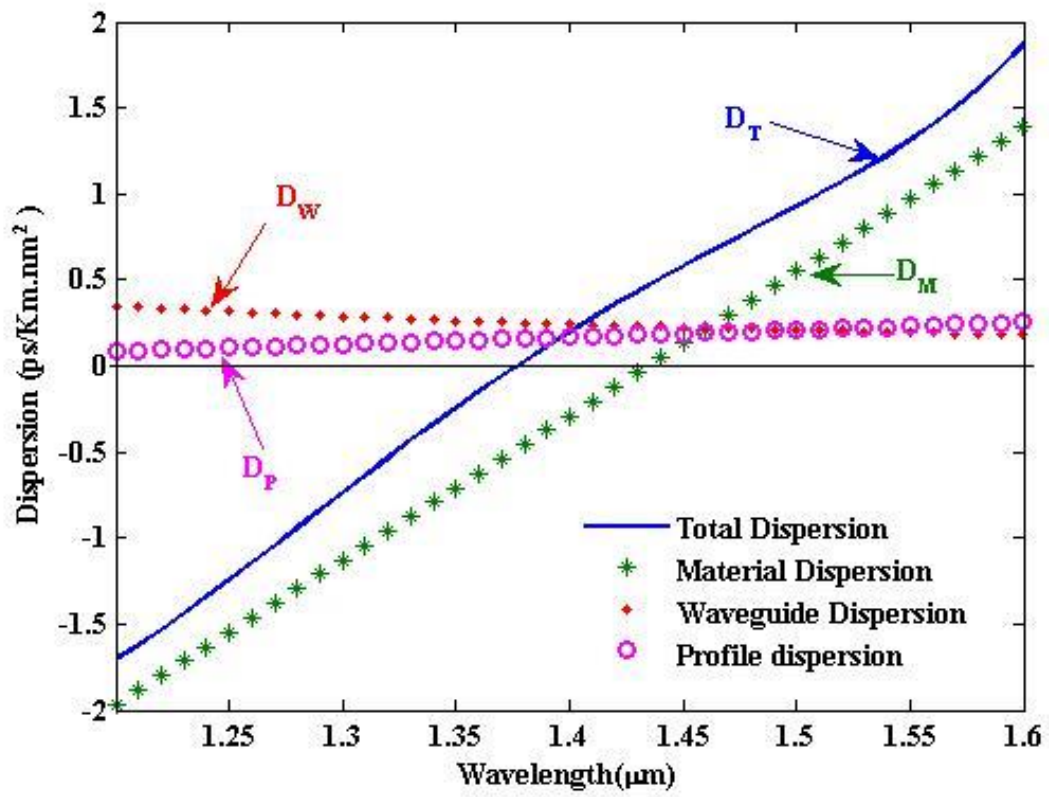

Figure (10) The relation between the three types of dispersion \& wavelength.

In figure 10 shows the three types of dispersion and the sum of total dispersion in equation (15) with wavelength for the optical fiber $\mathrm{K}_{3}$ and $\mathrm{K}_{4}$ with core radius $\mathrm{r}=4 \mu \mathrm{m}$.

2-To complete the study, we chose single-mode optical fiber $\left(\mathrm{K}_{3}\right.$ and $\left.\mathrm{K}_{4}\right)$ for core and cladding respectively $\Delta=0.0043$ at $1.55 \mu \mathrm{m}$ and core radius $(\mathrm{r}=3,4,5) \mu \mathrm{m}$ is noted. 
Figure 11 shows there is an increasing in the normalized propagation constant $\mathrm{b}$ by increasing the core radius.

Figure 12 shows there is a very small change in material dispersion by increasing the core radius and there is a small shift in zero-dispersion toward the longer wavelength.

Figure 13 by increasing the core radius, we noted an increase in waveguide dispersion, although the best sample with core radius $4 \mu \mathrm{m}$ (less amount of waveguide dispersion).

Figure 14 shows the increasing profile dispersion with wavelength. As the radius increases the wavelength decreases.

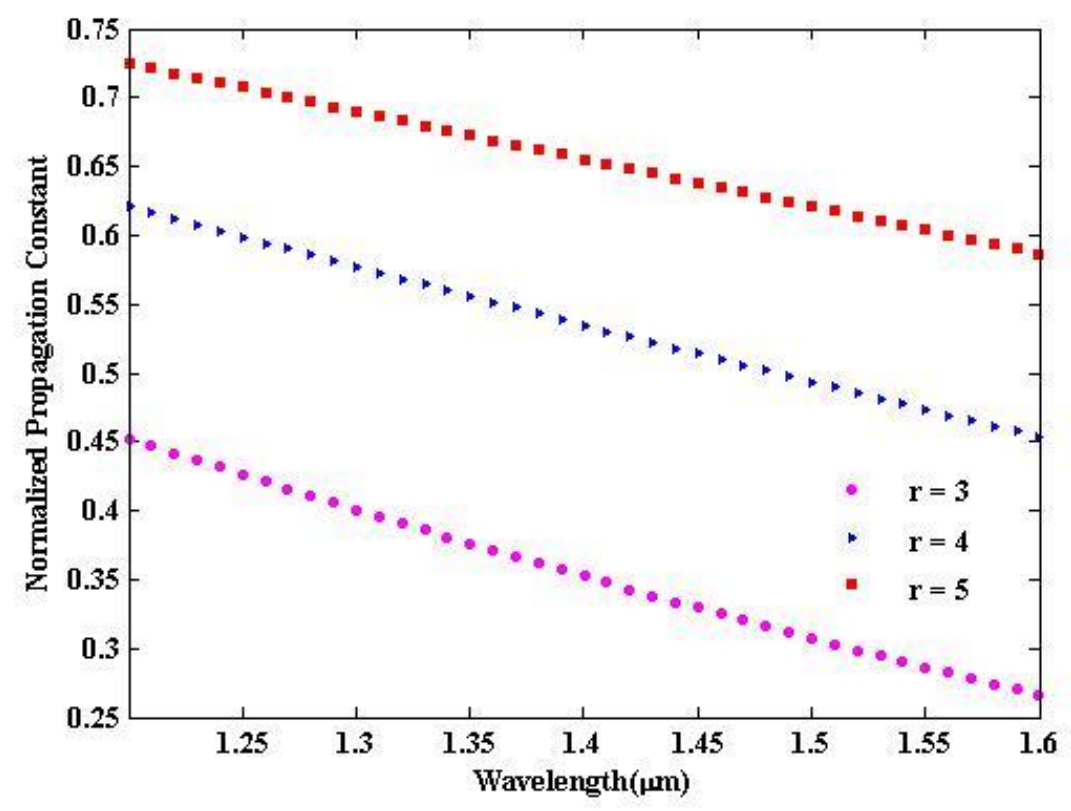

Figure (11) The relation between normalized propagation constant \& wavelength. 


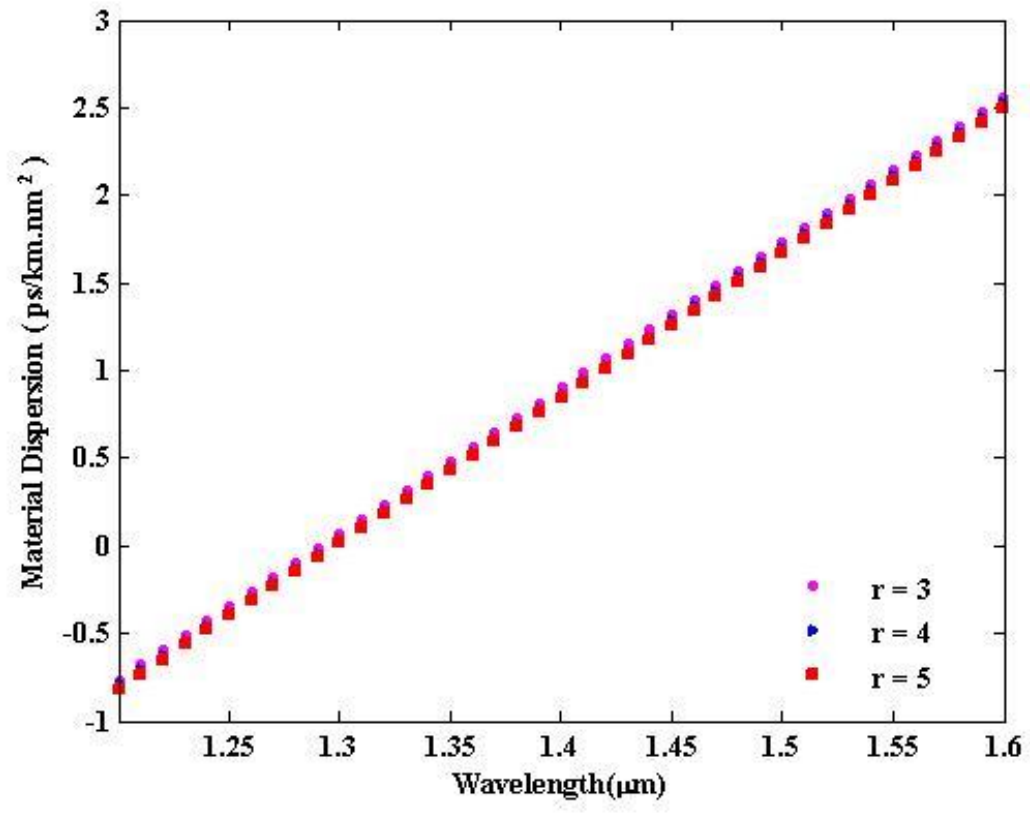

Figure (12) material dispersion in different radius.

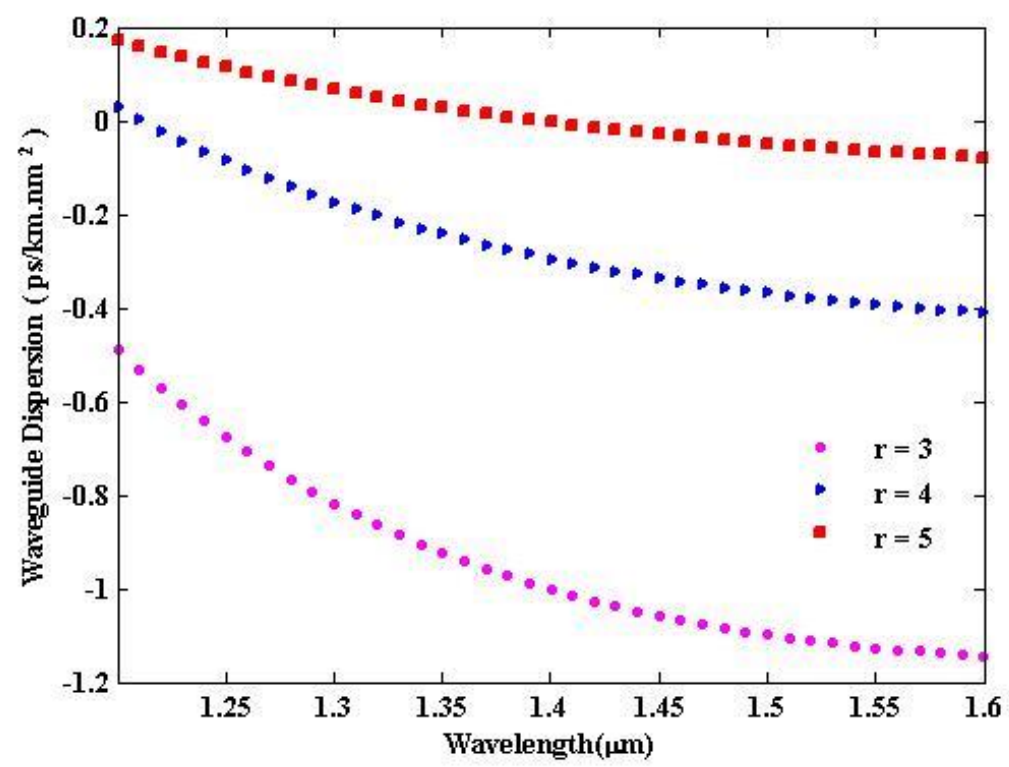

Figure (13) Waveguide dispersion in different radius. 


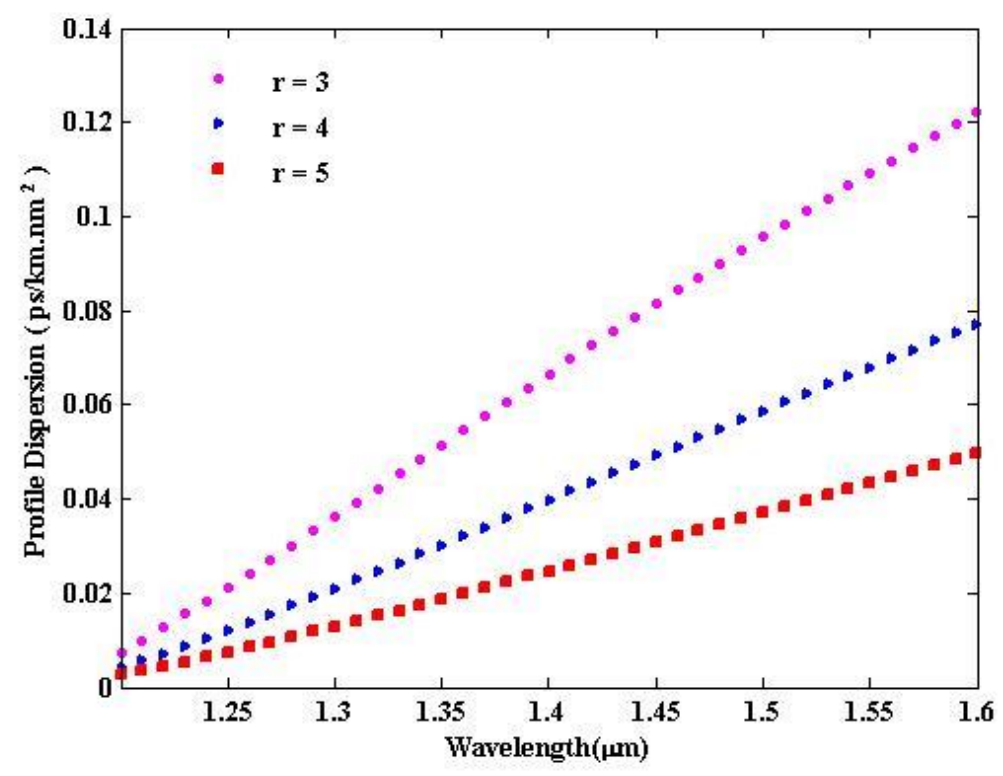

Figure (14) Profile dispersion in different radius.

\section{Conclusion}

The variation in relative refractive index exerts a strong influence on propagation within the singlemode optical fiber and has to be taken into account during the investigating dispersion. We have used three equation to calculate the types of dispersion, namely, material dispersion, waveguide dispersion, and profile dispersion.

Total dispersion has been obtained from the summation of these three types; it is also obtained from total dispersion expression in the range of wavelength between (1.2-1.6) $\mu \mathrm{m}$.

The effect of variation in the core diameter of single-mode optical fiber has been also investigated. It can be noted that the normalized propagation constant is increased by increasing the relative refractive index. While the propagation constant shifts to higher normalized frequency as the relative refractive index increased.

As the refractive indices of the core and cladding are decreased the Y parameter is increased.

There is a small chanage in material and waveguide dispersion with variation of relative refractive index, while the waveguide dispersion significantly affected by vartaion. relative refractive index.

The normalized propagation constant of single-mode optical fiber increased with increasing the core diameter.

There is a small chanage in material dispersion due to increasing core diameter, while the two athers (waveguide and profile dispersion) show by increasing cor dimeter and the profile dispersion decreasing by increasing the core diameter. 
The study of the filed through the layers of the optical fiber and investigation of mode field dimater can be proposed as future work of this study.

\section{Reference}

1. Agrawal, G.P., Fiber-optic communication systems. Vol. 222. 2012: John Wiley \& Sons.

2. Senior, J.M. and M.Y. Jamro, Optical fiber communications: principles and practice. 2009: Pearson Education.

3. Mohammed, S.Q. and A.M.A.M. Al-Hindawi, Study of Optical Fiber Design Parameters in Fiber Optics Communications. Kurdistan Journal of Applied Research, 2017. 2(3): p. 302-308.

4. Kumar, S. and M.J. Deen, Fiber optic communications: fundamentals and applications. 2014: John Wiley \& Sons.

5. Dutton, H.J., Understanding optical communications. Vol. 1. 1998: Prentice Hall PTR Durham, North Carolina, USA.

6. Keiser, G., Optical fiber communications. Wiley Encyclopedia of Telecommunications, 2003.

7. Buck, J.A., Fundamentals of optical fibers. Vol. 50. 2004: John Wiley \& Sons.

8. Woodward, B. and E.B. Husson, Fiber optics installer and technician guide. 2006: John Wiley \& Sons.

9. Crisp, J., Introduction to fiber optics. 2005: Elsevier.

10. Gambling, W., H. Matsumura, and C. Ragdale, Mode dispersion, material dispersion and profile dispersion in graded-index single-mode fibres. IEE Journal on Microwaves, Optics and Acoustics, 1979. 3(6): p. 239-246.

11. Raghavendra, M. and P. Prasad, A novel approach for optimized dispersion in optical fiber communication. International Journal of Research and Reviews in Applied Sciences IJRRAS, 2010. 4. 
12. Mussina, R., et al. Numerical modeling method for the dispersion characteristics of single-mode and multimode weakly-guiding optical fibers with arbitrary radial refractive index profiles. in Physics and Simulation of Optoelectronic Devices XXI. 2013. International Society for Optics and Photonics. 